\title{
Better together? Regression analysis of complex survey data after ex-post harmonization
}

Anna-Carolina Haensch ${ }^{1}$ and Bernd Weiss ${ }^{2}$

\section{Keywords}

survey weight, meta-analysis, survey harmonization, multi-level

\footnotetext{
${ }^{1}$ University of Mannheim, Germany

${ }^{2}$ GESIS Leibniz Institute for the Social Sciences, Germany
}

Corresponding author:

Anna-Carolina Haensch, University of Mannheim, Mannheim, Germany

Email: ahaensch@mail.uni-mannheim.de 


\section{Introduction}

What can researchers do if information from a single data source is insufficient to investigate a particular research question? An increasing number of researchers then pool, (ex-post) harmonize and analyze survey data from different survey providers for their research questions (SDR 2020; IPUMS 2020; CLOSER 2020; MAELSTROM 2020; MTUS 2020). They aim to study heterogeneity between groups over a long period, pick up subtle differences between groups or examine small subgroups. All these are potential research strategies that lead researchers to combine and harmonize survey data from different providers.

In this context, harmonization refers to procedures aimed at improving the comparability of different surveys and measures (Granda, Wolf and Hadorn 2010). Both ex-ante and ex-post harmonization have a strong tradition in cross-cultural surveys. Ex-ante harmonization refers to harmonization during survey design and before data collection. Ex-post harmonization to harmonization after data collection. While the harmonization of variables is already a complex task (Granda et al. 2010, 331), the analysis of complex survey data after variable harmonization is not necessarily easier.

Complex surveys are surveys that incorporate a complex sampling design. They are widely used in the social sciences, and ex-post harmonization projects will most likely also include surveys with complex sampling design. Ignoring the complex sampling design can lead to biased population inferences not only in population means and shares but also in regression coefficients (DuMouchel and Duncan 1983; Pfeffermann and Sverchkov 2009). For regression models widely used in quantitative sociological research, analysts often try to account for the sampling design through additional predictors. With ex-post survey harmonization, this often becomes impossible since it could require the analyst to harmonize many other predictors. Besides, these predictors might not be observed for several of the surveys, creating severe missing data problems. Another possible limitation would be that the sampling variables are not suitable for the analysis due to their associations with focus variables (Lumley 2010, 105). However, there is a second option to account for the complex sampling design: it is possible to estimate a survey-weighted regression. In this article, we explore this second option in the context of harmonized complex survey data and compare different approaches for regression analyses of survey data after ex-post harmonization and how to incorporate survey weights and survey weighting.

The idea of combining or pooling data has also surfaced in areas other than sociology and cross-cultural studies. The terms to describe the general idea of combining data differ widely between disciplines and even projects; terms used include, for example, individual person data (IPD) meta-analysis (MA) in medicine and psychology mainly (Riley, Lambert and Zaid 2010; Burke, Ensor and Riley 2017), mega-analysis (Boedhoe et al. 2019) or just merely describing the strategy as "pooling raw data" (Korn and Graubard 1999). The IPD MA literature, in particular, proposes two general approaches for the regression analysis of pooled raw data: (1) synthesizing regression coefficients estimated from the single data sets (two-stage approach) or (2) estimating a regression on the combined data sets (one-stage approach). We study which method is more suited for the analysis of complex survey data after ex-post harmonization. We draw on the methodological literature on IPD meta-analysis and related research on combining data from rolling or periodic sampling (Kish 1979; Kish and Verma 1986; Kish 1999) and analyzing data from complex cross-cultural surveys (Joye, Sapin and Wolf 2019). We show that the distribution of survey weights in the respective data set will play an important role when applying these two meta-analytical approaches. We demonstrate that unless the coefficient of variance for the survey weights is small, the assumption of known within-study variances for two-stage analysis is problematic and can result in biased point estimates, while the one-stage analysis remains unaffected.

Our article starts with an introduction to IPD MA and its two main approaches. Also, we give an overview of survey-weighted regression. We then use Monte Carlo simulations to evaluate the performance of different meta-analytical approaches. We also exemplify the difference between two-stage and one-stage approaches with a real-world example about same-sex couples and family satisfaction in Germany.

\section{Regression analysis of complex survey-based data after ex-post harmonization}

\section{Overview}

Two approaches for IPD meta-analyses For regression analyses of complex survey-based data, we will draw on two different analysis approaches developed for individual participant data (IPD) meta-analysis, one-stage and two-stage meta-analysis.

These distinct ways of conducting an IPD MA have emerged over the last few years. The more common approach, which is also closer to a more classical (aggregate person data) meta-analysis, is the so-called two-stage approach. Meta-analysts 
first analyze each of the $k=1, \ldots, K$ studies separately to obtain study-specific effect sizes. They then combine the $K$ independent effect sizes by calculating a weighted (often inverse error-variance based) average. The second approach is the so-called one-stage IPD MA approach. Here the combined data is analyzed simultaneously. This flexible approach requires further modeling decisions like the possible inclusion of separate intercepts for different surveys or the addition of random intercepts or slopes. These one-stage analyses with random effects are special cases of hierarchical or multi-level models (Simmonds et al. 2005).

Regarding the comparability of these two approaches, one-stage and two-stage approaches often lead to similar estimates of treatment effects (Olkin and Sampson 1998; Stewart et al. 2012). However, one-stage analyses improve the power of detecting effects for continuous and binary data (Lambert et al. 2002; Simmonds et al. 2005). Power is also higher for detecting nonlinear relationships between continuous treatment and outcome and the discovery of treatment-covariate interactions if researchers use one-stage approaches (Simmonds and Higgins 2007). Burke et al. (2017) give an overview of previous results and a list of ten key reasons why one-stage and two-stage approaches may lead to different results in practice. We will add another reason to that list, connected to the variances of survey weights.

Literature Review Few authors have tackled the topic of combining complex survey data from an (ex-post) survey harmonization or (IPD) meta-analytical perspective. Roberts and Binder (2009) briefly discuss the two main meta-analytical approaches, one-stage, and two-stage meta-analysis, in the context of survey data. They stress to either frame the research question in a design based or model-based way. Fox (2011) proposes a methodological framework for combining survey data and a comparison of this framework with the one by Cochran (1997) for the combination of experiments. Korn and Graubard (1999) suggest methods to adjust survey weights when pooling surveys with different numbers of observations. Besides, survey methodologists and statisticians have combined data from surveys though they generally do not refer to it as meta-analysis. For instance, Leslie Kish focused on combining data from rolling or periodic samples and non-overlapping probability samples from the same population (Kish 1979; Kish and Verma 1986; Kish 1994, 1999).

Prospectively planned pooled analyses such as extensive international cross-cultural studies are usually not called metaanalyses, although they also are a method for summarizing the evidence. Joye et al. (2019) discuss the pooling and weighting of surveys in the context of harmonized cross-cultural studies. Following Kish, statisticians have tackled different types and aspects of pooling data. While Cochran (1997) and Fuller and Burmeister (1972) concentrated on combining sub-populations, Kalton and Anderson (1986) and Skinner and Rao (1996) among others worked on the topic of multiple frames for the same target population. Lohr and Raghunathan (2017)) and Fox (2011) provide overviews over other related topics like dual-frame problems, statistical matching, combining survey data with data from other surveys, small-area-estimation or re-weighting data with several samples.

As a next step, we look at possible analytical options for combining complex survey data after survey harmonization. Afterward, we explore the performance of these approaches in our simulation of weighted pooled survey data.

\section{Two-stage IPD meta-analytical approaches}

We start by presenting the two-stage (meta-analytical) approach for analyzing pooled data. Suppose each study $k(k=1, \ldots, K)$, that we want to include into our analysis, has $n_{k}$ observations $i\left(i=1, \ldots, n_{k}\right)$. We are interested in the effect of a single independent variable on the dependent variable. A simple approach would be to estimate regressions in each study separately and then, in the second stage, to combine the effect sizes obtained in the first stage (Burke et al. 2017, 856).

If we follow this approach, we estimate regression coefficients for every survey. We can either estimate a weighted or an unweighted estimate (DuMouchel and Duncan 1983). Likelihood inference is the standard estimation approach in most software and also in the survey package in R (Lumley 2016) that will be used in the following. In case of the incorporation of inverse-probability weights, this leads to a pseudolikelihood (for details see Rabe-Hesketh and Skrondal 2006; Binder 1983; Pfeffermann 1993; Pfeffermann and Sverchkov 2009).

After estimating the regression coefficients for the different surveys, we reach the second stage of the two-stage analysis (Burke et al. 2017, 857). In this stage, we combine the regression coefficients, similar to a classical meta-analysis. If we assume between-study homogeneity, the weight of each survey point estimate depends solely on the variance of its estimate (a so-called fixed-effect model). 
Lumley and Scott (2017, 268/269) discuss estimating variances of the weighted regression coefficients. We use the recommended sandwich delta-method (White 1982; Binder 1983), which is implemented in the survey package for generalized linear models. Researchers should note that it does not acknowledge the weighting's random component (if the weighting is not solely by design). In large samples, this random component can be ignored.

In some cases, it may be reasonable to assume that a common effect exists (for example, medical studies using the same treatment for a very similar group of patients, measuring the same outcome, etc.) and estimating a fixed-effect (FE) model. However, such an assumption of homogeneity can rarely be made for most studies (Viechtbauer 2007), especially in the social sciences. Studies are likely to have systematic differences, e.g., between measurements used (Elliot, Raghunathan and Schenker 2018). With strong study heterogeneity, random-effects (RE) models are considered more appropriate for the second stage of the analysis. The underlying assumption is that there is not one common effect for all the studies, but instead, one assumes a distribution of effects across studies.

When calculating the combined RE estimate, we again take an inverse-variance weighting approach, but we now incorporate an estimate of the between study-variance $\hat{\tau}^{2}$ (Borenstein et al. 2009, section 2).

There are many methods of estimating $\hat{\tau}^{2}$, for example, the method of moments estimator of DerSimonian and Laird (1986, 2015), also called DL estimator. For continuous outcomes, Veroniki et al. (2016, 55) advocate using REML estimation as a preferable alternative to the DL estimator, which we also use in the following simulations.

After covering the basics of the two-stage approach, we now move on to one-stage analysis. A one-stage analysis is more complicated but also more flexible.

\section{One-stage IPD meta-analytical approaches}

When conducting a one-stage analysis, we first combine surveys and then analyze the combined data set. An abundance of modeling options characterizes the one-stage (IPD meta-analytical) approach. For all included predictors, separate fixed or random study effects can be included.

We start with the simplest model, the one-stage model without separate fixed or random study effects. Let us still assume we have $K$ studies for the IPD MA with $n_{k}\left(i=1, \ldots, n_{k}\right)$ observations each. We are interested in the effect of the independent variable $X$ on the dependent variable $Y$.

1. One-stage model without separate fixed study effects (linear model):

$$
y_{i k}=\alpha+\beta x_{i k}+e_{i k}, e_{i k} \sim N\left(0, \sigma^{2}\right)
$$

Here we treat the data as if they came from one large survey. We do not model study heterogeneity. However, it is generally not recommended to treat the combined studies as one big survey, ignoring the clustered structure. Researchers instead include fixed or random study effects for that reason (see the next models).

2. One-stage model with separate fixed study effects:

Adding a separate fixed intercept term $\alpha_{k}$ per study allows for different survey means in the dependent variable (when controlling for the independent variable(s)) and the clustering of observations in studies (Burke et al. 2017, 859).

$$
y_{i k}=\alpha_{k}+\beta x_{i k}+e_{i k}, e_{i k} \sim N\left(0, \sigma^{2}\right)
$$

It is also possible to allow varying slopes for the surveys by adding interaction effects of survey indicators and independent variables. This can be done for one or more surveys in the meta-analysis.

3. One-stage model with one or more random study effects:

The separate fixed study effects for the intercept can also be replaced with a random effect, e.g., when one is interested in the baseline intercept $\alpha$ or in a measure of between-study heterogeneity (Burke et al. 2017, 859). Another advantage is that we reduce the number of parameters compared to the fixed effects model (instead of estimating a fixed effect for each survey, we only estimate two parameters $\alpha$ and $\tau_{\alpha}^{2}$ ). One should note that the inclusion of random effects demands a sufficiently high number of included surveys (Snijders and Bosker 1993).

$$
y_{i k}=\alpha_{k}+\beta x_{i k}+e_{i k}, e_{i k} \sim N\left(0, \sigma^{2}\right), \alpha_{k} \sim N\left(\alpha, \tau_{\alpha}^{2}\right) .
$$


As in the fixed effect case, it is possible to add random study effects for the independent variables, i.e., random slopes, too. The random slope model allows the explanatory variable to have a different effect for each study. One can also assume a separate residual variance per study. Readers should be aware that the inclusion of weights into multi-level models like the RE one-stage model here is still an unresolved research question (see Asparouhov (2006), Carle (2009)). For more modeling, estimation and software options in the context of one-stage (IPD meta-)analysis see the articles by Debray et al. (2015) and Burke et al. (2017).

\section{Survey weights and the use of survey weights in regression analysis}

Design, nonresponse and post-stratification weights In this section, we very briefly introduce the three types of survey weights most widely used in practice: design weights, nonresponse weights, and post-stratification weights. For the calculation of a design weight for an observation $i$; we use the inverse of the probability $p_{i, \text { selection }}$ to be selected (Horvitz and Thompson 1952 and Lumley 2010,4): $w_{i}=\left(p_{i, \text { selection }}\right)^{-1}$. Nonresponse weights are closely related to sampling weights. Units are

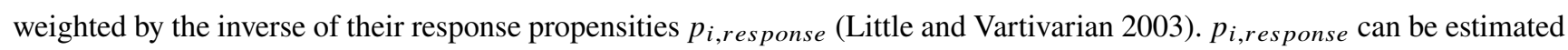
if researchers have information about respondents and non-respondents as in the European Social Survey (Blom 2009, 29). There is ongoing research about the use of such nonresponse weights (Blom 2009; Kreuter et al. 2010; Krueger and West 2014).

Many surveys (Little and Vartivarian 2005, 161) do not provide design weights or nonresponse weights but instead poststratification or calibration weights. Post-stratification weights are used to adjust the sample to known population totals (Lumley 2010, 136). Post-stratification is also called cell weighting or adjustment weighting. This weighting approach can be considered when the distribution of auxiliary variables (e.g., sex, age groups) is known in the population and differs from those in the sample. Post-stratification grows more complicated with the number of post-stratification variables. If the joint distribution of the variables is not known but only the marginal distributions, it is necessary to use alternative closely related techniques like calibration (Deville and Särndal 1992). These challenges and the procedures used for calibration (e.g., raking) are not the focus of this article; for an overview, see Lumley $(2010,139)$.

Survey-weighted regression analysis - Hybrid models To gain familiarity with the topic of survey-weighted regression, we will briefly examine survey-weighted regressions with just one data set in this section. We then move on to the case with pooled complex survey data. For the moment, we concentrate on three simple cases: (1) a simple linear regression meeting the Gauss-Markov assumptions and observations drawn with exogenous sampling, i.e., independent from the error term (2) a simple linear regression where we are faced with endogenous sampling, also called selection on unobservables and (3) a linear heterogeneity of effects model where data points have different sampling probabilities depending on the groups. While in the first case, the use of weights is usually discouraged; it can be beneficial in the second and third cases.

Case 1: We start with a simple linear regression meeting the Gauss-Markov assumptions and exogenous sampling. We are interested in the regression of a variable $Y$ on $X$. For the moment, we only have data from one survey. Let us assume that the population from which this survey was drawn has different strata $s$, and the population units have differing sampling probabilities depending on the strata $s$. The coefficient $\beta$ is the same in all strata. The error term has a normal distribution with mean 0 and variance $\sigma^{2}, e$, and the strata are independent. Since the sampling is only dependent on the strata, it is also independent of the error term $e$ and exogenous. Like the sampling probabilities, the means of $X$ may differ per strata (see Figure 1 (a)).

However, the Gauss-Markov (GM) assumptions are still met and thus there is no reason to prefer the weighted estimate to the ordinary unweighted estimate. As an example, the unweighted OLS estimate $\hat{\beta}$ is already approximately unbiased and has minimum variance among all linear approximately unbiased estimators (DuMouchel and Duncan 1983, 536). While the weighted coefficients are also approximately unbiased, they are not the best linear approximately unbiased estimates (BLUE), whereas the OLS estimates are (Verbeek 2004, 16-17). Let us now move on to cases where weights are indeed needed for approximately unbiased estimates.

Case 2: Again, we want to estimate a simple linear regression of $Y$ on $X$. However, this time the sampling is endogenous, e.g., depending on the unobservable error term. For this reason, endogenous sampling is also called selection on unobservables. Estimates not weighted for the endogenous sampling are biased (Solon, Haider and Wooldridge 2013). 
A classic example of endogenous sampling is oversampling, which depends on the covariates of the substantial model (the independent variables) and the dependent variables and, therefore, the error term. For example, a researcher attempts to estimate a regression model of family income on years of education. If high-income families with a low number of years of schooling were oversampled compared to middle and low-income families with the same number of years, then the error term is correlated with the sampling probabilities, and the regression estimates will be biased. We have visualized this situation in Figure 2 (a). Less transparent points represent higher sampling probabilities. One can easily see that the regression line estimated without weights will have a higher intercept than the population regression line in red. Plus, in the case of heterogeneous variance for the error term, endogenous sampling can shift the slope estimate as well (see Figure 2 (b)).

In the case of endogenous sampling, one needs to use inverse-probability weights to achieve consistent estimates (Solon et al. 2013, 17). However, we have to note that estimating nonresponse or post-stratification weights correcting for all the bias is highly unrealistic. The bias reduction depends on two associations: (1) on the correlation between the response propensity and the response propensities predictors and (2) on the correlation between the error term and the response propensities predictors (Little and Vartivarian 2003). Unfortunately, these correlations are often not very high (Kreuter et al. 2010, 405).

Case 3: Last but not least, we want linear heterogeneity of effects model where data points have different sampling probabilities depending on the groups (see Figure 1 (b)).

It is not possible to give general recommendations for which estimate, unweighted $\hat{\beta}$ or weighted $\hat{\beta}_{w}$, to use when assuming a model with heterogeneous coefficients. Neither $\hat{\beta}$ nor $\hat{\beta}_{w}$ are generally approximately unbiased estimates of the average coefficient $\bar{\beta}$ (DuMouchel and Duncan 1983, 537). Solon et al. $(2013,19)$ show that there are two reasons why the unweighted estimate does not identify the average population effect $\bar{\beta}$. The first is easy to understand. Let us assume that the effect of children on partnership quality is more negative in urban areas than in rural areas. If we oversample rural areas, we would not expect the unweighted estimate of the average coefficient to be the same as the average population effect. This is the first source of bias in case of an unweighted estimate. But Solon et al. $(2013,20)$ provide another reason why the OLS estimate is not consistent. Extreme values of the independent variables can have a particularly large influence on the estimates. Therefore, the unweighted average also depends on the difference in the within-strata variance of the independent variables. The weights deal with the first source of bias but not the second. If the within-variances are equal in all strata, WLS is thus consistent, and OLS is not. As Solon et al. $(2013,20)$ notice, this is the "knife-edge special case" and not true in general. They recommend comparing weighted and unweighted estimates to get an idea of possible bias.

Adding additional covariates to the model would be another possibility to account for nonresponse or oversampling and remove bias (Pfeffermann and Sverchkov 2009, 461). However, the covariates might not be available in some cases, but only the weights (Solon et al. 2013, 15-17). The inclusion of additional covariates might complicate the interpretation of focus model parameters (Sterba 2009, 727), for an example see also Pfeffermann and Sverchkov (2009, 463). Also, in the case of harmonized surveys, conditioning on observed selection/nonresponse variables will be especially difficult since they are likely to differ between surveys. Therefore, we strongly prefer hybrid models that are primarily model-based and account for disproportionate selection through weighting instead of conditioning on all complex sampling features (see Sterba (2009) for a comparison of the model-based, design-based, and hybrid framework).

Conclusion Out of the three cases we covered - (1) a simple linear regression meeting the Gauss-Markov assumptions and with exogenous sampling, (2) a simple linear regression where we are faced with endogenous sampling, and (3) a linear heterogeneity of effects model with different sampling probabilities depending on strata - we only need to use survey-weighted regressions in the latter two. In the first case, survey weighting only leads to an inflation of the variance of the regression coefficients. However, whether a study falls under (1) is usually uncertain and therefore recommended to compare weighted and unweighted estimates.

Survey-weighted regression analysis - The meta-analytical case With pooled data sets, it is easy to see that the need for survey weighting in case of endogenous sampling and heterogeneity of effects models (or a combination of these problems) does not simply vanish in the meta-analytical case. To make this explicit, let us remember that each data set is first analyzed separately in two-stage models. If these models' unweighted coefficients are biased, the combined coefficient will be biased, too (except in the unlikely case in which these biases cancel each other out).

For practical applications, this still leaves us with the question if we even need to use survey weights due to nonresponse or effect heterogeneity. Bollen et al. (2016) provides a review of various diagnostic tests used to determine if survey weights are 
necessary for regression analysis. These tests can be divided into two groups: the first group of tests examines the difference between weighted and unweighted regression coefficients; the second one whether, conditional on the independent variable (Pfeffermann 1993; Asparouhov and Muthen 2007), the dependent variable, and the weights are correlated (DuMouchel and Duncan 1983; Fuller 2009; Pfeffermann and Sverchkov 1999). However, one should note that these tests were developed for OLS/WLS regression with a single data set.

Plus, it is uncertain and unlikely that all the surveys fall into the case of exogenous sampling and no effect heterogeneity. This problem is especially pressing with real complex surveys. Surveys can have multiple aspects of over-sampling across various stages, or it is unclear whether the outcome variables are exogenous. We recommend comparing weighted and unweighted estimates in all cases to get an idea of possible bias.

\section{A comparison of one-stage and two-stage approaches in case of pooled complex survey data}

\section{Introduction to the simulation design}

After having gained an overview of different (meta-analytical) strategies and survey-weighted regressions, we will now further explore the use of survey weights in the context of regression analysis of complex survey data after ex-post harmonization. We look at practical problems and questions that arise when conducting a regression analysis with weighted observations after pooling: Do survey-weighted one-stage and two-stage analysis perform differently? Is it possible to include random effects into the survey-weighted analysis, especially if we have to assume study heterogeneity?

When evaluating the performance of different analytical approaches, researchers, in general, look at the bias, the variance, the root mean square error (RMSE), and the coverage of these approaches. While bias, variance, and RMSE should be as small as possible, coverage should be around 0.95 in the case of standard 95\%-confidence intervals. In our case, it is cumbersome to derive these four measures analytically for our multitude of approaches. Therefore, we turn to Monte Carlo simulations. We focused on relatively simple simulation scenarios, to attribute any differences in performance to the (meta-analytical) method, rather than getting caught up in complexities of the data. An overview of the data settings, the implemented sampling procedures, and the meta-analytical approaches we used are given in Tables A1 and A2.

Surveys are homogeneous, except for Simulation Nr. 3 and 4. In these, we extensively covered the inclusion of random effects to model study heterogeneity.

\section{Methodological decisions and differences between one-stage/two-stage meta-analysis}

State of the Art In the following, we focus on the differences between the two general approaches - one-stage vs. two-stage analysis. All in all, one-stage meta-analyses are more flexible in accommodating different assumptions, and they offer the more exact likelihood specification. Furthermore, two-stage meta-analytical results are easily biased when few studies and few participants/events are at hand (Burke et al. 2017, 863). In these cases, bias arises since the assumptions that the study effects have a normal sampling distribution and that their variances are known cannot be upheld (Stijnen, Hamza and Özdemir 2010).

We will now add another new reason why in practice, a one-stage analysis might outperform a two-stage analysis. We will demonstrate in our next simulation that with a moderately high coefficient of variance for the weights, the assumption of known within-study variances is highly problematic and can result in biased point estimates.

Simulation and Results In this section, we conduct two different simulations. We use two different heterogeneity of slope models for data generation. While the sampling is endogenous for both of them, we change the range of sampling probabilities, therefore changing the variance in the inverse-probability weights. In Simulation Nr. 1 (see Table A1), the coefficient of variation $C V$ for the weights amounts to ca. 0.76. This $C V$ value is not unrealistic; it corresponds to the coefficient of variation for the weights in the second wave of the German family survey 'Familiensurvey' (Deutsches Jugendinstitut (DJI) 2003). The coefficient of variation $C V$ for the weights in Simulation Setting Nr. 2 is smaller, it is only half this size.

Examining the results and comparing one-stage and two-stage meta-analytical results, we see that our weighted two-stage slope estimates are biased in case of a high CV, whereas the weighted one-stage estimates are approximately unbiased (see Figure 3). The two-stage results are only approximately unbiased, when - all other things being equal - we have a low variance for the weights. 
To understand the mechanism behind this non-intuitive result, we take a closer look at the single-survey estimates and their estimated variances. If we look at the distribution of the survey-weighted error terms from several surveys, we see that some of the surveys have bumps at negative values of the error term (see Figure 4 (a)).

This happens when observations with low inclusion probability are included. Since these observations have very high inverse-probability weights, they also drive up the variance estimate. Simultaneously, point estimates are lower in these cases, since the highly influential observations drag the point estimate down. If we now weight the point estimates by their variance estimates in the second stage, the point estimates which are smaller and have larger variance estimates get a lower (meta-analytical) weight than the larger point estimates with smaller variance estimates (see Figure 4 (b)). Therefore, we overestimate the coefficient in our two-stage meta-analysis. The extent of the bias will depend on the correlation between the added variance due to the weights and the point estimates. Providing corrections of the variance is difficult. If we used our knowledge that the variance before weighting is equal for all surveys in our simulation, we could get an approximately unbiased estimate. Still, in real-world applications, we do not know the distribution between the two variance sources.

Fox $(2011,128)$ also notes that the weighting procedure leads to a higher variance of the estimates. She proposes adjusting the meta-analytical weight by the design effect. Kish (1965) showed that the design effect can be decomposed into the variation in weights (unequal weighting effect) and the component that is attributable to the stratification/clustering of data (stratification/clustering effect). We only want to correct for the variation in weights. However, as Lê, Brick and Kalton (2002) noted, the canonical decomposition of the design effect provided by Kish (1965) is not a good approximation when weights are post-stratified or calibrated. Moreover, the decomposition depends on the assumption of constant within-strata (within-survey) variances, a potentially unrealistic assumption.

Instead of correcting the two-stage meta-analytical weights, we recommend using a one-stage meta-analysis, especially in the case of a high coefficient of variation for the weights. E.g., a high coefficient of variation for the weights will often occur in the case of post-stratification with many sparsely occupied cells. However, we acknowledge that this might not always be possible, e.g., if researchers want to include information on strata/primary sampling units into their model (Rabe-Hesketh and Skrondal 2006). This would further complicate a one-stage meta-analytical model and is an important argument in favor of two-stage models.

\section{Transformations of survey weights}

We have investigated the general questions of when to use survey weights in analyses with pooled complex survey data. Our study also demonstrated that a one-stage meta-analysis offers some advantages. We will now concentrate on study heterogeneity, which requires special attention when including survey weights in a one-stage meta-analysis.

We take a look at study homogeneity/heterogeneity and introduce a transformation needed in the case of study heterogeneity. We speak of study homogeneity when all studies in a meta-analysis were undertaken in the same way with the same measurement, target population, etc. However, we will seldom be able to assume study homogeneity. If this assumption is violated, e.g., the studies used different measurements for the same construct, or the target population differs slightly between surveys, we have to deal with and model so-called study heterogeneity (Viechtbauer 2007).

Study heterogeneity is a problem since reflecting the heterogeneity of the studies in our analysis may be complex and challenging. However, it is often crucial since variables and target populations will somewhat differ between included surveys. A conventional approach is to use a model with random effects for the surveys. This can be done regardless of whether we estimate a one-stage or two-stage analysis. Estimating a two-stage analysis with random effects or including a random effect in a one-stage analysis is equivalent to assuming that the true coefficient is not the same for all studies. Instead, the true coefficient has a distribution over surveys. Researchers often choose a normal distribution for this distribution (Burke et al. 2017, 859).

Unfortunately, the use of random effects is tricky in the case of a weighted one-stage analysis. It is known that weights cannot be used in their 'raw' form in weighted multilevel/hierarchical analysis since the point and standard error estimates will be biased (Asparouhov 2006, 442, 445). Asparouhov (2006), building on earlier work by Pfeffermann et al. (1998), recommended two methods 'A' and 'B' (see Equation 4 and 5) for transforming the weights (Asparouhov 2006, 443). 
Method 'A' scales the weights so that the transformed weights sum to the cluster sample size (in our case the survey sample size $\left.n_{k}\right)$ :

$$
w_{i k}^{*}=w_{i k}\left(\frac{n_{k}}{\sum_{i} w_{i k}}\right) .
$$

Method 'B' scales the weights so that the transformed weights sum to the effective sample size:

$$
w_{i k}^{*}=w_{i k}\left(\frac{\sum_{i} w_{i k}}{\sum_{i} w_{i k}^{2}}\right) .
$$

'A' and 'B' become equivalent when cluster sizes and cluster sample sizes are constant (Asparouhov 2006, 445). Method ' $A$ ' seems to be better suited if we want to examine regression coefficients, and method ' $\mathrm{B}$ ' is recommended when we are interested in estimates of heterogeneity (Carle 2009, 10).

Asparouhov $(2006,444)$ stressed that in the weighted multi-level analysis, we only need to use level-1 weights. This is because level-1 (individual observations) and level-2 (clusters/surveys) weights appear in different places in the pseudo maximum likelihood (Carle 2009, 2). In our case, we exclusively have level-1 weights in a one-stage analysis, since the studies do not have weights in a one-stage analysis and we do not have to worry about separating level-1 and level-2 weights.

We should, however, check if the ratio between the weights from different studies is meaningful, that is, to be interpreted in the usual sense of oversampling (Asparouhov 2006, 444). E.g., if observation A in survey A has a weight of two and observation B in survey B a weight of four, the probability with which A was drawn should be half of B's probability. As demonstrated in Korn and Graubard (1999), this is generally not the case if we have different numbers of survey observations for surveys drawn from the same population, and these survey weights are not standardized around one. In these cases, it will be necessary to standardize the weights using the simplest transformation that Korn and Graubard (1999, see Equation 8.2-6 on page 283) proposed. However, one should again be aware of the assumption of a common population in this case. Researchers should be able to describe this population. The weights after transformation sum up to the population size and can be interpreted in the usual sense of oversampling.

Multilevel models that incorporate survey weights use a pseudo maximum likelihood (PML) approach, and the transformed weights $w_{i k}^{*}$ are included then into the PML (see Rabe-Hesketh and Skrondal 2006, 806). Carle (2009) compares a variety of software programs and software implementations as for example Stata's gllamm. Statas mixed command fits linear mixedeffects models and offers both transformation methods (options 'size' for method ' $A$ ' and 'effective' for method 'B'). In $R$ there is a workaround with the function scale_weights from the sjstats-package (Luedecke 2018). The function scale_weights offers both transformation methods 'A' and 'B' recommended by Asparouhov (2006). After transforming the weights, the researcher then can include these weights as frequency weights in the lmer-function from the package lme4 (Bates et al. 2015). The lmer-function then uses a penalized least squares method for maximum likelihood fitting. Another critical point is that even Carle $(2009,3)$ and Asparouhov (2006) concede that the transformation 'A' and 'B' are not ideal and recommend analyzing with both scaling methods and then comparing the results.

Simulation and Results We conducted the two simulations Nr. 3 and 4 to explore modeling study heterogeneity and the inclusion of random effects in a weighted one-stage analysis. In Simulation Nr. 3, we created 25 artificial populations, each with a different intercept. These finite populations come from a theoretical super-population. Each finite population has five strata. These five strata have different intercept components in the data generating model (but are equal over all populations). The sampling probability also differs by strata. We draw an equally sized survey from each of the populations. What is crucial is that we now have study heterogeneity, which has to be reflected in the meta-analytical model. In this simulation, we compare the weighted one-stage analysis, which does not model the study's heterogeneity (no random effect), and different one-stage analysis incorporating either a random intercept or a random slope. Of course, we also compare the two transformation approaches (Method 'A' and 'B').

Moving on to the results for the first simulation with heterogeneous intercepts between studies (see Table 1), we see that the RMSE for the intercept (the intercept now differs between super-populations) is slightly better for the weighted one-stage MA with RE than without RE. The intercept coverage is above 0.95 for all weighted approaches. However, the coverage is below 0.9 for the slope of the one-stage RE model (which does not differ between super-populations). We could not find differences between the two transformation methods for our case (Method 'A' and 'B', see Equation 4 and 5). This was to be expected since our study sample sizes are constant. 
What is intriguing is that we cannot determine a difference in bias between the unweighted and weighted estimates for the between-study standard deviation estimate $\tau$ (see Figure 5). This is because the super-population distribution for the intercept is independent of the sampling procedure in our case. However, remember that the weights are still needed for approximately unbiased point estimates.

Social-science researchers are often interested in estimating the influence of one variable on another and are therefore often concentrating on slope estimates. Consequently, we created another simulation (Nr. 5), where we again created 25 artificial populations but now with a different slope per study instead of a different intercept.

The results (see Table 2) are similar to the first simulation. When we include a random effect for the slope into our meta-analysis, the coverage for the slope is very high, but not satisfying for the common intercept. Including an RE for the intercept instead of a slope RE does not solve the problem; the slope coverage is meager.

To sum up, better results for the parameters affected by study heterogeneity go hand in hand with less preferred results for common homogeneous parameters, if one includes RE into the meta-analytical model.

\section{Practical example: Same-sex couples and their satisfaction with family life in Germany}

We will conduct a brief example using data from three surveys in Germany. We are interested in the satisfaction with family relationships for people in a same-sex relationship versus other peoples in relationships. Previous research is not conclusive on whether a person in same-sex relationships experiences family relationships differently than other persons in relationships and/if people in same-sex couples potentially experience unique stressors regarding their family relationships (Cramer and Roach 1988; Willoughby et al. 2008; Baiocco et al. 2014; Lampis et al. 2020).

Our example will use German survey data to examine family satisfaction of same-sex and opposite-sex couples from three German surveys. The data sets used are the first wave of the pairfam panel, the second wave of the Generations and Gender survey (Gauthier et al. 2018), and the wave "ba" of the Socio-economic Panel (SOEP). The German Family Panel pairfam ("Panel Analysis of Intimate Relationships and Family Dynamics") was launched in 2008 and is a multi-disciplinary, longitudinal study for researching partnership and family dynamics in Germany (Brüderl et al. 2017). The Generations and Gender Survey (GGS) is a longitudinal study intended to provide information about the relationships between children and their parents and relationships in couples (Ruckdeschel et al. 2006). The German Socio-Economic Panel (SOEP) is a longitudinal panel that started in 1984 and includes questions on household composition, occupation, employment, earnings, health, and life satisfaction (Goebel et al. 2019).

These surveys all included questions on (1) the sex of the current partner (which allowed to identify people in same-sex and opposite-sex relationships) and (2) satisfaction with family life (in case of the GGS they asked after the satisfaction with the relationship to various family members whose average we took for the satisfaction with the family). The surveys/panel wave data were all conducted between 2008 and 2010.

We do not have direct information on the sexual orientation of respondents. However, as already mentioned, we can infer who is living in a same-sex partnership since respondents were asked for their partner's sex. Concerning the legal situation in Germany, in 2008/2010 (and since 2001), same-sex couples could enter a civil partnership but not marriage. This lasted until 2017, when marriage was also opened for same-sex couples in Germany.

Our target population is people in Germany older than the age of 15 who are either married, in a civil partnership, or have been in a partnership for longer than six months (LATs or cohabitation). We will reflect the subsampling (people in relationships) in the variance estimation of the survey-weighted regression (West, Berglund and Heeringa 2008). We also reflect the fact that while the original target populations of the three survey are largely overlapping between the GGS (people in Germany aged 20-83) and SOEP (people in Germany older than 16), pairfam has a particular population (cohorts born 1971-73, 1981-83, and 1991-93, ergo 15-17, 25-27 and 35-37 years old).

We will mirror the various overlaps through composite factors that are also often used in dual-frame surveys for weighting. We used the composite factor based on the numbers of observations of the various studies proposed by (Xia et al. 2010), which is very similar to the transformation Korn and Graubard (1999) recommends for raw weights from different surveys. 
Concerning the two-stage analysis, in the first stage, we estimate simple linear regressions of the family satisfaction regressed on the same-sex partnership indicator (and an intercept) for each survey separately. We use a fixed-effect (metaanalytical) model for the second stage (with the estimated regression coefficients from the first stage as effect estimates) since the number of surveys is low.

The one-stage analysis model has three separate intercepts for each of the three different surveys and the same-sex partnership indicator as independent variables. We used the survey package (Lumley 2016) for the weighted estimation of the one-stage model and the first stage of the two-stage models.

The estimated (meta-analytical) coefficients can be seen in Table 3, the estimated coefficients for the three surveys in the first stage of the two-stage approach are available in the appendix (see Tables A3, A4 and A5). We see quite a big difference between the two estimates. The two-stage model is strongly driven towards 0 by the estimate from the GGS, which is not significantly different from 0 . Since its standard error is of the same order as those of SOEP and pairfam, it greatly influences the two-stage model. Of course, survey weighting is not the only source of difference between the one-stage and two-stage coefficients (see Burke et al. (2017)). Factors that also (could) influence the estimates are the clustering of estimates in surveys, the specification of residual variances, and the "unbalanced" samples (regarding the proportion of same-sex couples). However, the coefficient of variation is also non-negligible, $77 \%$ for the GGS, for pairfam $85 \%$ and $106 \%$ for the SOEP (after correcting for the overlap).

After this applied real-world example, we will now conclude with our most important results and conclusions.

\section{Discussion}

\section{Results}

We have answered several crucial questions about the inclusion of survey weights in the regression analysis of complex surveys after ex-post harmonization. We first dealt with the problem of when survey weights should be included in the analysis. Survey weights are required for approximately unbiased estimates in the case of endogenous sampling. They are also helpful in the case of heterogeneity of effects models when strata differ not only in their sampling probability but also their coefficients. However, weights can also increase the variance of estimates.

Researchers should also be aware that nonresponse or post-stratification weights have to be highly correlated with the sampling/inclusion probability to reduce statistical bias (Little and Vartivarian 2003; Kreuter et al. 2010). These correlations will seldom be large in practice. Therefore, the weights will only mitigate part of the bias.

Another crucial topic is the difference between the one-stage and two-stage approaches. Burke et al. (2017) gave ten general recommendations for the use of one-stage or two-stage IPD meta-analyses and reasons why results may differ between the two methods. We added another one to that list: If we have a medium to high coefficient of variation of the survey weights, the assumption of known within-study variances cannot be upheld for the two-stage meta-analytical approach. Two-stage meta-analytical estimates will be biased.

Afterward, we directed our attention to study characteristics like study size and study homogeneity/heterogeneity. Another point that we covered is the inclusion of random effects in a one-stage analysis. Carle (2009) and Asparouhov (2006) demonstrated that even the performance of RE models with transformed weights is not always optimal. We confirmed this observation.

\section{Limitations and Future Research}

The question of using weights will concern many analyses in the social sciences with survey data. In our simulations, we explored linear regressions since researchers are most familiar with these models. More research has to be conducted to check if conclusions that we have drawn for linear models are also applicable to other models from the class of generalized linear models, e.g., logistic regressions. Another interesting class of models would be survival models, e.g., proportional hazards models.

We also only briefly covered the topic of the inclusion of weighted one-stage meta-analysis with random effects. We were able to draw valuable lessons from the neighboring field of weighted multi-level analysis. However, optimal scaling methods are not yet available (Asparouhov 2006; Carle 2009) for weighted RE models like one-stage RE analysis. A comparison of the different software implementations is another topic to be covered. 
Last but not least, we only covered the use of survey weights to avoid bias. Weighting is, however, also used in regressions to correct for heteroscedasticity and improve efficiency. Plus, we did not cover replicate weights, which are often provided instead of survey weights in order to guarantee data confidentiality. Replicate weights also allow computing jackknife variance estimates. The use of weights to improve efficiency in meta-analyses would be yet another research question.

\section{Conclusions}

This article has conducted first explorations into the field of weighted analysis of pooled complex survey data. We have identified several settings where survey weights are needed to achieve approximately unbiased estimates. Most intriguingly for practitioners, we found differences in performance between the two main (meta-)analytical approaches - one-stage and two-stage analysis. In a two-stage analysis, bias can be introduced through the weighting procedure. Fortunately, by avoiding the step of estimating point and variance estimates for the single surveys, a weighted one-stage analysis remains approximately unbiased. Even though we recommend using the one-stage analysis approach in the case of weighted complex survey data, researchers should take care when pooling the data. Transformation will be required if studies are heterogeneous. Plus, incorporating PSUs/strata information in a one-stage analysis may prove very difficult. And as in other analyses, choosing an appropriate model remains of high importance.

\section{REFERENCES}

Asparouhov, Tihomir. 2006. "General Multi-Level Modeling with Sampling Weights.” Communications in Statistics - Theory and Methods 35:439-60.

Asparouhov, Tihomir and Bengt Muthen. 2007. "Testing for Informative Weights and Weights Trimming in Multivariate Modeling with Survey Data.” Proceedings, Section on Survey Research Methods, American Statistical Association.

Roberto Baiocco, Lilybeth Fontanesi, Federica Santamaria, Salvatore Iovern, Barbara Marasco, Emma Baumgartner, Brian L. B. Willoughby and Fiorenzo Laghi. 2014. "Negative Parental Responses to Coming Out and Family Functioning in a Sample of Lesbian and Gay Young Adults.” Journal of Child and Family Studies 24:1490-1500.

Bates, Douglas, Martin Mächler, Ben Bolker and Steve Walker. 2015. "Fitting Linear Mixed-Effects Models Using lme4.” Journal of Statistical Software 67:1-48.

Binder, David A. 1983. “On the Variances of Asymptotically Normal Estimators from Complex Surveys.” International Statistical Review / Revue Internationale de Statistique 51:279-92.

Blom, Annelies G. 2009. "Measuring, Explaining and Adjusting for Cross-Country Differences” in Unit Nonresponse: What Can Process Data Contribute? A Thesis submitted for the Degree of Doctor of Philosophy in Applied Social and Economic Research, Institute for Social and Economic Research. University of Essex.

Boedhoe, Premika S. W., Martijn W. Heymans, Lianne Schmaal, Yoshinari Abe, Pino Alonso, Stephanie H. Ameis, Alan Anticevic, Paul D. Arnold, Marcelo C. Batistuzzo, Francesco Benedetti, Jan C. Beucke, Irene Bollettini, Anushree Bose, Silvia Brem, Anna Calvo, Rosa Calvo, Yuqi Cheng, Kang Ik K. Cho, Valentina Ciullo, Sara Dallaspezia, Damiaan Denys, Jamie D. Feusner, Kate D. Fitzgerald, Jean-Paul Fouche, Egill A. Fridgeirsson, Patricia Gruner, Gregory L. Hanna, Derrek P. Hibar, Marcelo Q. Hoexter, Hao Hu, Chaim Huyser, Neda Jahanshad, Anthony James, Norbert Kathmann, Christian Kaufmann, Kathrin Koch, Jun Soo Kwon, Luisa Lazaro, Christine Lochner, Rachel Marsh, Ignacio Martínez-Zalacaín, David Mataix-Cols, José M. Menchón, Luciano Minuzzi, Astrid Morer, Takashi Nakamae, Tomohiro Nakao, Janardhanan C. Narayanaswamy, Seiji Nishida, Erika L. Nurmi, Joseph O'Neill, John Piacentini, Fabrizio Piras, Federica Piras, Y. C. Janardhan Reddy, Tim J. Reess, Yuki Sakai, Joao R. Sato, H. Blair Simpson, Noam Soreni, Carles Soriano-Mas, Gianfranco Spalletta, Michael C. Stevens, Philip R. Szeszko, David F. Tolin, Guido A. van Wingen, Ganesan Venkatasubramanian, Susanne Walitza, Zhen Wang, Je-Yeon Yun, Paul M. Thompson, Dan J. Stein, Odile A. van den Heuvel and Jos W. R. Twisk. 2019. "An Empirical Comparison of Meta- and Mega-Analysis With Data From the ENIGMA Obsessive-Compulsive Disorder Working Group". Frontiers in Neuroinformatics, 12.

Bollen, Kenneth A., Paul P. Biemer, Alan F. Karr, Stephen Tueller and Marcus E. Berzofsky, 2016. “Are Survey Weights Needed? A Review of Diagnostic Tests in Regression Analysis". Annual Review of Statistics and Its Application 3:375-92.

Borenstein, Michael, Larry V. Hedges, Julian P. T. Higgins and Hannah R. Rothstein. 2009. "Introduction to Meta-Analysis". Chichester, U.K: Wiley. 
Josef Brüderl and Karsten Hank, Johannes Huinink, Bernhard Nauck, Franz J. Neyer, Sabine Walper, Philipp Alt, Elisabeth Borschel, Petra Buhr, Laura Castiglioni, Stefan Friedrich, Christine Finn, Madison Garrett, Kristin Hajek, Michel Herzig, Bernadette Huyer-May, Rüdiger Lenke, Bettina Müller, Timo Peter, Claudia Schmiedeberg, Philipp Schütze, Nina Schumann, Carolin Thönnissen, Martin Wetzel and Barbara Wilhelm. 2017. The German Family Panel (pairfam). Technical Report ZA5678 Data file Version 8.0.0, GESIS Data Archive, Cologne.

Burke, Danielle L., Joie Ensor and Richard D. Riley 2017. "Meta-analysis Using Individual Participant Data: One-stage and Two-stage Approaches, and Why They May Differ". Statistics in Medicine 36:855-75.

Carle, Adam C. 2009. "Fitting Multilevel Models in Complex Survey Data with Design Weights: Recommendations". BMC Medical Research Methodology 9(49):1-14.

CLOSER (2020). The Home of Longitudinal Research. Retrieved June 20, 2020 (https://www. closer .ac.uk/).

Cochran, William G. 1997. Sampling Techniques. A Wiley publication in Applied Statistics. 3rd. Hoboken, NJ: Wiley.

David W. Cramer and Arthur J. Roach. 1988. "Coming Out to Mom and Dad: A Study of Gay Males and Their Relationships with Their Parents". Journal of Homosexuality 15:79-92.

Debray, Thomas P. A., Karel G. M. Moonsand, Gert van Valkenhoef, Orestis Efthimiou, Noemi Hummeland, Rolf H. H. Groenwold, Johannes B. Reitsma and GetReal Methods Review Group. 2015. "Get Real in Individual Participant Data (IPD) Meta-analysis: A Review of the Methodology.” Research Synthesis Methods 6:293-309.

DerSimonian, Rebecca and Nan Laird. 1986. Meta-analysis in Clinical Trials. Controlled Clinical Trials 7:177-88.

DerSimonian, Rebecca and Nan Laird. 2015. “Meta-analysis in Clinical Trials Revisited.” Contemporary Clinical Trials 45:139-45.

Deutsches Jugendinstitut (DJI) (2003). Wandel und Entwicklung familialer Lebensformen - 3. Welle (Familiensurvey). München. ZA3920 Datenfile Version 1.0.0.

Deville, Jean-Claude and Carl-Erik Särndal. 1992. “Calibration Estimators in Survey Sampling.” Journal of the American Statistical Association 87:376-82.

DuMouchel, William H. and Greg J. Duncan (1983). "Using Sample Survey Weights in Multiple Regression Analyses of Stratified Samples.” Journal of the American Statistical Association 78:535-43.

Elliott, Michael R., Trivellore E. Raghunathan and Nathaniel Schenker. 2018. Combining Estimates from Multiple Surveys. Pp. 1-10 in Wiley StatsRef: Statistics Reference Online. American Cancer Society.

Fisher, Ronald A. 1922. "On the Mathematical Foundations of Theoretical Statistics." Philosophical Transactions of the Royal Society of London A: Mathematical, Physical and Engineering Sciences 222:309-368.

Fox, Karla. 2011. “A Framework for the Meta-analysis of Survey Data.” PhD dissertation. Department of Mathematics and Statistics. Queen's University, Kingston, Ontario, Canada.

Fuller, Wayne A. 2009. Sampling Statistics. Hoboken, NJ: Wiley-Blackwell.

Fuller, Wayne A. and Burmeister, Leon F. 1972. Estimators for Samples Selected from Two Overlapping Frames. Proceedings of the Social Statistics Section, American Statistical Association.

Granda, Peter, Christof Wolf, and Reto Hadorn. 2010. Harmonizing Survey Data. Pp. 315-32 in Survey Methods in Multinational, Multiregional, and Multicultural Contexts, edited by J. A. Harkness, M. Braun, B. Edwards, T. P. Johnson, L. Lyberg, P. Ph. Mohler, B.-E. Pennell and T. W. Smith. Hoboken, NJ: John Wiley \& Sons.

Horvitz, Daniel G. and Donovan J. Thompson. 1952. "A Generalization of Sampling Without Replacement from a Finite Universe." Journal of the American Statistical Association 47:663-85.

IPUMS. 2020. Integrated Public Use Microdata Series. Retrieved June 20, 2020 (https://www .maelstrom-research.org/).

Joye, Dominique, Marlène Sapin and Christof Wolf. 2019. "Weights in Comparative Surveys? A Call for Opening the Black Box". Harmonization: Newsletter on Survey Data Harmonization in the Social Sciences 5:2-16.

Kalton, Graham and Dallas W. Anderson. 1986. "Sampling Rare Populations.” Journal of the royal statistical society. Series A (general) 149:65-82.

Kish, Leslie. 1965. Survey Sampling. Wiley Classics Library. Hoboken, NJ: Wiley.

Kish, Leslie. 1979. “Samples and Censuses.” International Statistical Review/Revue Internationale de Statistique 47:99-109.

Kish, Leslie. 1994. "Multipopulation Survey Designs: Five Types with Seven Shared Aspects.” International Statistical Review/Revue Internationale de Statistique 62:167-86. 
Kish, Leslie. 1999. Cumulating/Combining Population Surveys. Survey Methodology 25:129-38.

Kish, Leslie and Vijay Verma. 1986. Complete Censuses and Samples. Journal of Official Statistics 2:381.

Graubard, Barry I. and Edward L. Korn. 1999. Analyses Using Multiple Surveys. Pp. 278-303 in Analysis of Health Surveys, edited by Barry I. Graubard and Edward L. Korn. Hoboken, NJ: Wiley-Blackwell.

Kreuter, Frauke, Kirsten Olson, James Wagner, Ting Yan, Trena M. Ezzati-Rice, Carolina Casas-Cordero, Michael Lemay, Andy Peytchev, Robert M. Groves, Trivellore E. Raghunathan. 2010. Using Proxy Measures and other Correlates of Survey Outcomes to Adjust for Non-response: Examples from Multiple Surveys. Journal of the Royal Statistical Society: Series A (Statistics in Society) 173:389-407.

Krueger, Brian S. and Brady T. West. 2014. Assessing the Potential of Paradata and Other Auxiliary Data for Nonresponse Adjustments. Public Opinion Quarterly 78:795-831.

Lambert, Paul C., Alex J. Sutton, Keith R. Abrams and David Jones. 2002. A Comparison of Summary Patient-level Covariates in Meta-regression with Individual Patient Data Meta-analysis. Journal of Clinical Epidemiology 55:86 - 94.

Lampis, Jessica, Silvia De Simone and Christopher K. Belous. 2020. Relationship Satisfaction, Social Support, and Psychological Well-Being in a Sample of Italian Lesbian and Gay Individuals. Journal of GLBT Family Studies 0:1-14.

Lê, Thanh, J. Michael Brick, and Graham Kalton. 2002. Decomposing Design Effects. In Section on Survey Research, New York.

Little, R. and Vartivarian, S. 2003. On Weighting the Rates in Non-response Weights. Statistics in Medicine 22:1589-99.

Little, Rod J. A. and Sonya Vartivarian. 2005. Does Weighting for Nonresponse Increase the Variance of Survey Means? Survey Methodology, 31:4-11.

Little, Rod J. A. 1993. Post-Stratification: A Modeler's Perspective. Journal of the American Statistical Association, 88:1001-12.

Lohr, Sharon L. and Trivellore E. Raghunathan. 2017. Combining Survey Data with Other Data Sources. Statistical Science, 32:293-12.

Luedecke, Daniel. 2018. sjstats: Statistical Functions for Regression Models. R package version 0.18.0.

Lumley, Thomas. 2010. Complex Surveys. Hoboken, NJ: John Wiley and Sons.

Lumley, Thomas. 2016. survey: Analysis of Complex Survey Samples. R package version 3.32.

Lumley, Thomas and Alastair Scott. 2017. Fitting Regression Models to Survey Data. Statistcal Science 32:265-78.

MAELSTROM 2020. Maelstrom Research. Retrieved June 20, 2020 (https://www .maelstrom-research.org/).

MTUS. 2020. Multinational Time Use Study. Retrieved June 20, 2020 (https://www . timeuse.org/mtus/).

Olkin, Ingram and Allan R. Sampson. 1998. Comparison of Meta-analysis versus Analysis of Variance of Individual Patient Data. Biometrics 54:317-22.

Pfeffermann, Danny. 1993. The Role of Sampling Weights When Modeling Survey Data. International Statistical Review / Revue Internationale de Statistique 61:317-37.

Pfeffermann, Danny, Skinner, Chris J., Holmes, D. J., Goldstein, H. and Rasbash, J. 1998. Weighting for Unequal Selection Probabilities in Multilevel Models. Journal of the Royal Statistical Society. Series B (Statistical Methodology) 60:23-40.

Pfeffermann, Danny and Michail Sverchkov. 1999. Parametric and Semi-Parametric Estimation of Regression Models Fitted to Survey Data. Sankhyā: The Indian Journal of Statistics, Series B (1960-2002) 61:166-86.

Pfeffermann, Danny and Michail Sverchkov. 2009. Chapter 39 - Inference under Informative Sampling. Pp. 455 - 87 in Handbook of Statistics. Vol. 29 Sample Surveys: Inference and Analysis, edited by C.R. Rao. Elsevier.

Rabe-Hesketh, Sophia and Anders Skrondal. 2006. Multilevel Modelling of Complex Survey Data. Journal of the Royal Statistical Society: Series A (Statistics in Society), 169:805-27.

Riley, Richard D., Paul C. Lambert and Ghada Abo-Zaid. 2010. Meta-analysis of Individual Participant Data: Rationale, Conduct, and Reporting. BMJ, 340:c221.

Roberts, G. and Binder, D. 2009. Analyses Based on Combining Similar Information from Multiple Surveys. Pp. $2138-47$ in Proceedings. Section on Survey Research Methods. JSM 2009.

Ruckdeschel, Kerstin, Andreas Ette and Gert Hullen and Ingo Leven. 2006. Generations and Gender Survey: Dokumentation der ersten Welle der Hauptbefragung in Deutschland, Volume 121a of Materialien zur Bevölkerungswissenschaft. Bundesinstitut für Bevölkerungsforschung (BIB), Wiesbaden.

SDR. 2020. Survey Data Recycling Project. Retrieved June 20, 2020 (https://www . asc. ohio-state. edu/dataharmonization/).

Simmonds, Mark C. and Julian P. T. Higgins. 2007. Covariate Heterogeneity in Meta-analysis: Criteria for Deciding between Metaregression and Individual Patient Data. Statistics in Medicine, 26:2982-99. 
Simmonds, Mark C., Julian P. T. Higgins, Lesley A. Stewart, Jayne F. Tierney, Mike J. Clarke and Simon G. Thompson. 2005. Meta-analysis of Individual Patient Data from Randomized Trials: A Review of Methods Used in Practice. Clinical Trials, 2:209-17.

Skinner, Chris J. and Jon N.K. Rao. 1996. Estimation in Dual Frame Surveys with Complex Designs. Journal of the American Statistical Association 91:349-36.

Snijders, Tom A. B. and Roel J. Bosker. 1993. Standard Errors and Sample Sizes for Two-Level Research. Journal of Educational Statistics 18:237-59.

Solon, Gary, Steven J. Haider and Jeffrey Wooldridge. 2013. What Are We Weighting For? Working Paper 18859, National Bureau of Economic Research.

Sterba, Sonya. 2009. Alternative Model-Based and Design-Based Frameworks for Inference From Samples to Populations: From Polarization to Integration. Multivariate Behavioral Research 44:711-40.

Stewart, Gavin B., Douglas G. Altman, Lisa M. Askie, Lelia Duley, Mark C. Simmonds and Lesley A. Stewart. 2012. Statistical Analysis of Individual Participant Data Meta-Analyses: A Comparison of Methods and Recommendations for Practice. PLOS ONE, 7:1-8.

Stijnen, Theo, Taye H. Hamza and Pinar Özdemir. 2010. Random Effects Meta-analysis of Event Outcome in the Framework of the Generalized Linear Mixed Model with Applications in Sparse Data. Statistics in Medicine, 29:3046-67.

Verbeek, Marno. 2004. A Guide to Modern Econometrics. 2. ed., reprint. with corr. edition. Chichester, UK: Wiley.

Veroniki, Areti A., Dan Jackson, Wolfgang Viechtbauer, Ralf Bender, and Jack Bowden, Guido Knapp, Oliver Kuss, Julian P.T. Higgins, Dean Langan and Georgia Salanti. 2016. Methods to Estimate the Between-study Variance and its Uncertainty in Meta-analysis. Research Synthesis Methods 7:55-79.

Viechtbauer, Wolfgang. 2007. Accounting for Heterogeneity via Random-effects Models and Moderator Analyses in Meta-analysis. Zeitschrift für Psychologie/Journal of Psychology 215:104-21.

Goebel, Jan, Markus M. Grabka, Stefan Liebig, Martin Kroh, David Richter, Carsten Schröder and Jürgen Schupp. 2019. The German Socio-Economic Panel (SOEP), Jahrbücher für Nationalökonomie und Statistik 239:345-60.

Gauthier, Anne H., Susana L.F. Cabaço, Tom Emery. 2018. Generations and Gender Survey study profile, Longitudinal and Life Course Studies 9:456-65.

West, Brady T., Patricia Berglund and Steven G. Heeringa. 2008. A Closer Examination of Subpopulation Analysis of Complex-Sample Survey Data. The Stata Journal, 8:520-31.

White, Halbert. 1982. Maximum Likelihood Estimation of Misspecified Models. Econometrica 50:1-25.

Willoughby, Brian L. B., Nathan D. Doty and Neena M. Malik. 2008. Parental Reactions to Their Child's Sexual Orientation Disclosure: A Family Stress Perspective. Parenting 8:70-91.

Xia, Kanru, Pedlow, Steven and Michael Davern. 2010. Dual-frame weights (landline and cell) for the 2009 minnesota health access survey. American Statistical Association -Proceedings of the Survey Research Methods Section. Pp. 3912-3922. 


\section{Tables}

\begin{tabular}{lllllllll}
\hline & \multicolumn{3}{c}{ Intercept } & \multicolumn{3}{c}{ Slope } \\
& Bias & SE & RMSE & Cov. & Bias & SE & RMSE & Cov. \\
\hline One-stage weighted & 0.02 & 5.81 & 5.81 & 0.97 & 0.01 & 0.64 & 0.64 & 0.94 \\
One-stage RE (int.) w. A & 0.57 & 5.36 & 5.39 & 1.00 & 0.01 & 0.53 & 0.53 & 0.82 \\
One-stage RE (int.) w. B & 0.57 & 5.36 & 5.39 & 1.00 & 0.01 & 0.53 & 0.53 & 0.82 \\
\hline
\end{tabular}

Table 1. Performance measures. For simulation setting see Simulation Nr. 3 in Table A1. Description of all analysis models in Table A2.

\begin{tabular}{lllllllll}
\hline & \multicolumn{7}{c}{ Intercept } & \multicolumn{3}{c}{ Slope } \\
& Bias & SE & RMSE & Cov. & Bias & SE & RMSE & Cov. \\
\hline One-stage weighted & 0.07 & 2.73 & 2.73 & 0.95 & 0.01 & 0.43 & 0.43 & 0.96 \\
One-stage RE (int.) w. A & 0.07 & 2.73 & 2.73 & 0.93 & 0.04 & 0.42 & 0.42 & 0.65 \\
One-stage RE (int.) w. B & 0.07 & 2.73 & 2.73 & 0.93 & 0.06 & 0.43 & 0.43 & 0.63 \\
One-stage RE (slp.) w. A & 0.05 & 2.01 & 2.01 & 0.89 & 0.08 & 0.33 & 0.34 & 1.00 \\
One-stage RE (slp.) w. B & 0.05 & 2.01 & 2.01 & 0.89 & 0.08 & 0.33 & 0.34 & 1.00 \\
\hline
\end{tabular}

Table 2. Performance measures. For simulation setting see Simulation Nr. 4 in Table A1. Description of all analysis models in Table A2.

\begin{tabular}{lc}
\hline \multicolumn{1}{c}{ One-stage model, weighted observations } \\
\hline GGS Intercept & $8.41^{* * *}$ \\
& $(0.03)$ \\
pairfam Intercept & $8.67^{* * *}$ \\
& $(0.03)$ \\
SOEP Intercept & $7.85^{* * *}$ \\
& $(0.04)$ \\
Same-sex Partnership & $-\mathbf{0 . 8 1}{ }^{* * *}$ \\
& $(\mathbf{0 . 2 3})$ \\
\hline Num. obs. & 17579 \\
\hline & \\
\hline Two-stage model only coefficient for SSP shown), weighted observations \\
\hline Same-sex Partnership & $-\mathbf{0 . 4 0}{ }^{* *}$ \\
& $(\mathbf{0 . 1 7})$ \\
\hline${ }^{* * * *} p<0.001,{ }^{* *} p<0.01,{ }^{*} p<0.05$ &
\end{tabular}

Table 3. One-stage and two-stage meta-analysis with data from from GGS wave 3, pairfam wave 1, SOEP wave "y" (In all three surveys subsamples were taken: persons in partnerships). 


\section{Figures}

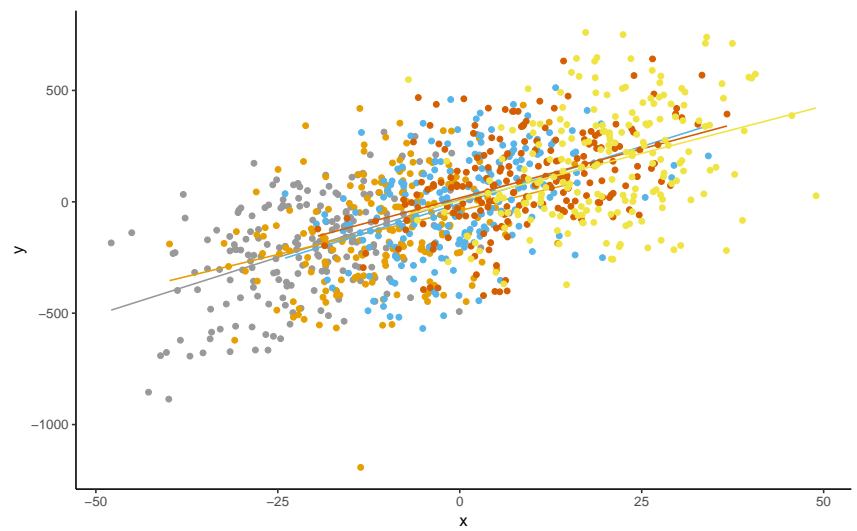

(a)

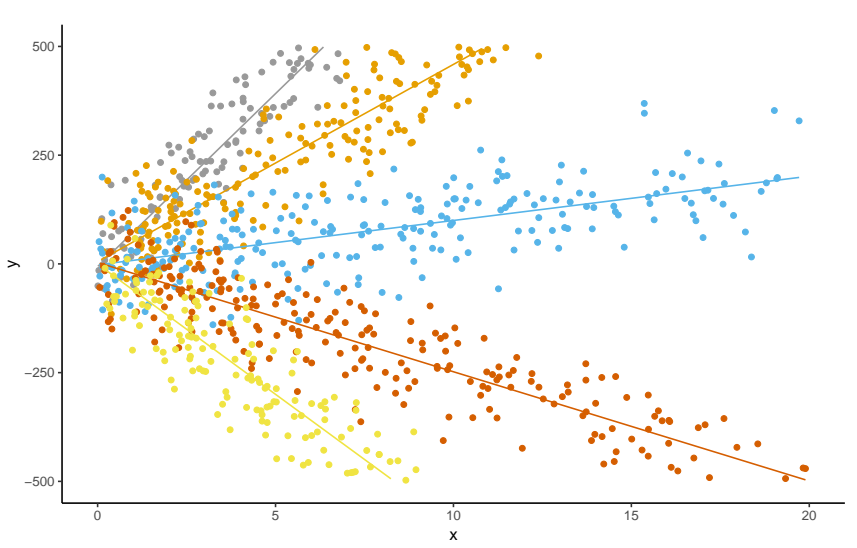

(b)

Figure 1. (a) Exemplary data setting with different means per strata (altered colors) but same data-generating model for the dependent variable. (b) Exemplary data setting with different slopes per strata. Points with the same color belong to the same strata.

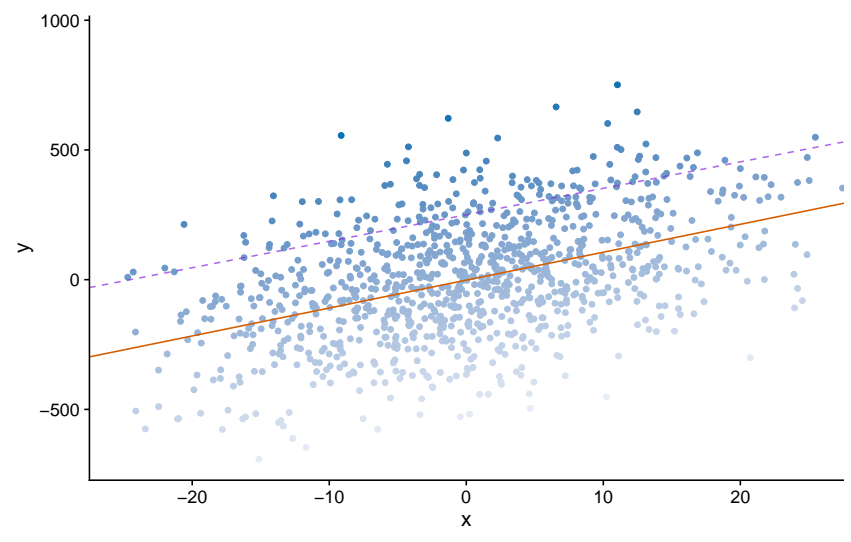

(a)

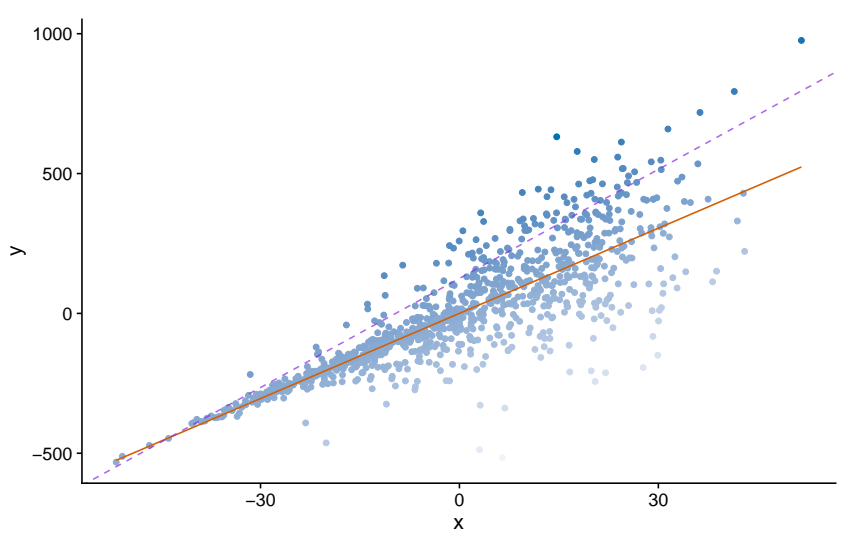

(b)

Figure 2. (a)Exemplary data setting with heterogeneous $Y$. Less transparent points represent higher sampling probabilities. The solid line is the regression line of $Y$ on $X$ in the population. The dashed line is the estimated unweighted regression line for a sample drawn with unequal sampling probabilities. (b) Exemplary data with different endogenous sampling probabilities. Less transparent points represent higher sampling probabilities. The solid line is the regression line of $Y$ on $X$ in the population. The dashed line is the estimated unweighted regression line for a sample drawn with unequal sampling probabilities.

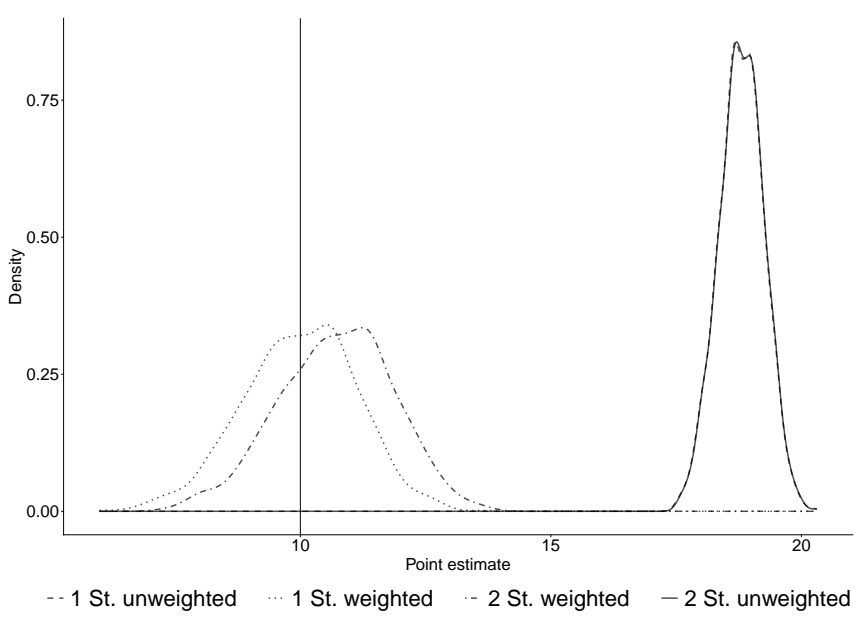

Figure 3. Density plot of slope point estimates. 1000 Monte Carlo repetitions. For simulation setting see Simulation Nr. 1 in Table A1. 


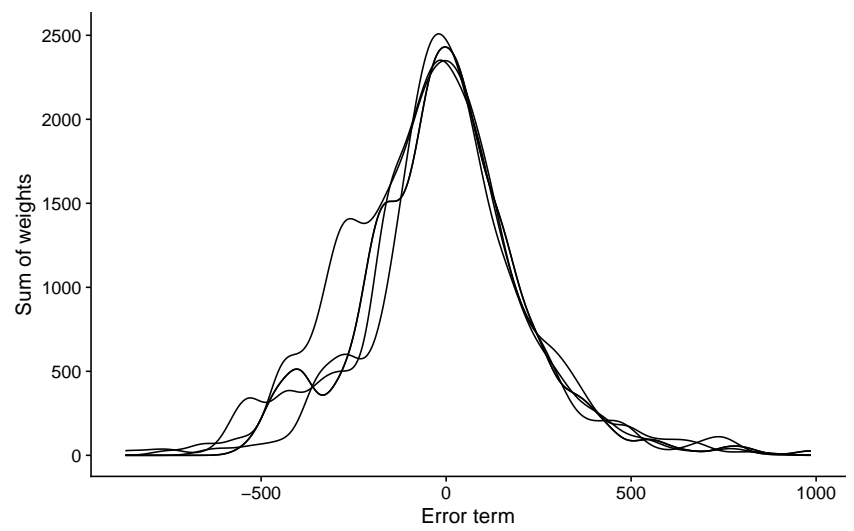

(a)

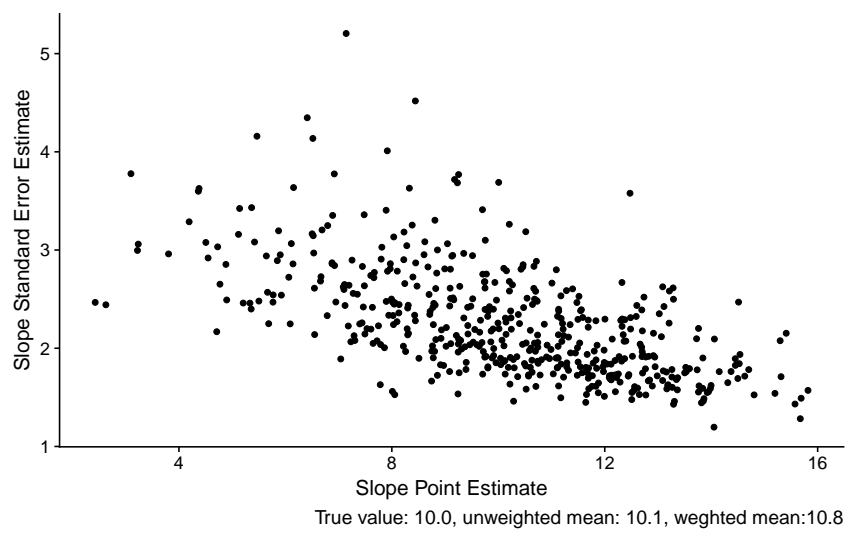

(b)

Figure 4. (a) Kernel 'density' plot of weighted error terms for five different surveys. The area under the curves sum up to the population size. For simulation setting see Simulation Nr. 1 in Table A1. (b) Scatter plot of slope point estimates against standard error estimates for 500 surveys. For simulation setting see Simulation Nr. 1 in Table A1.

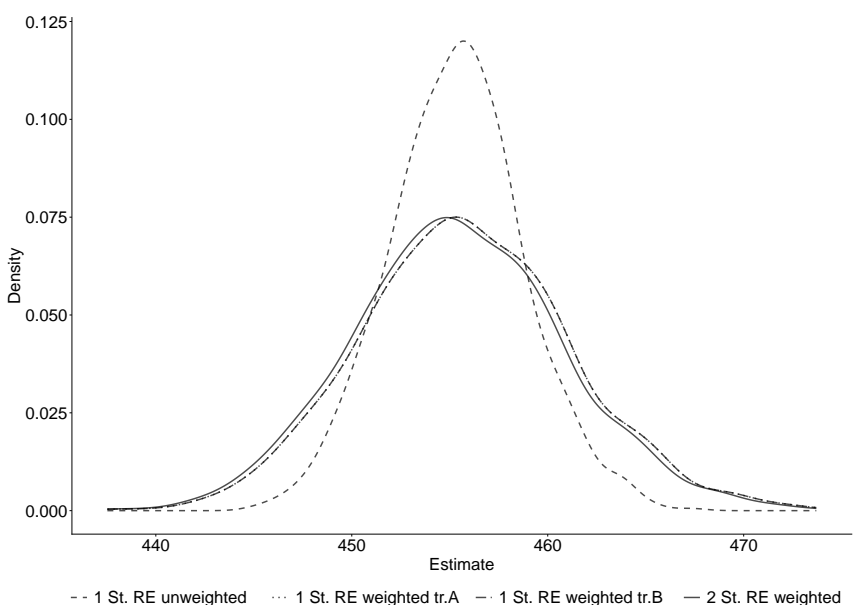

Figure 5. Density plot for between-survey intercept deviation estimate $\tau$. For simulation setting see Simulation Nr. 3 in Table A1. Description of all analysis models in Table A2. 
Appendix 


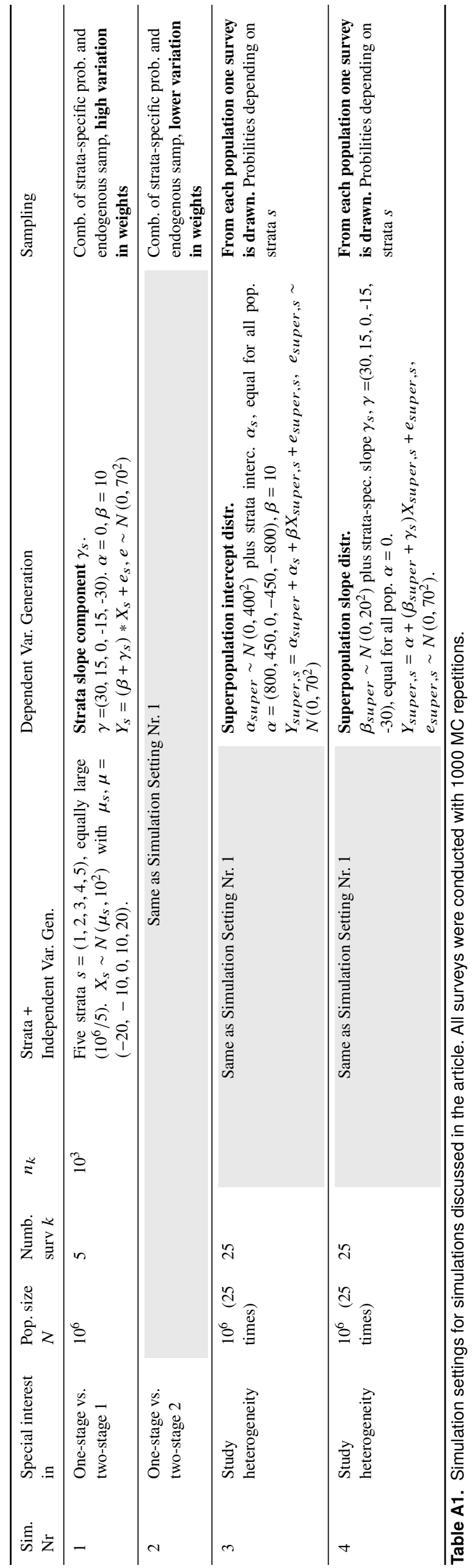




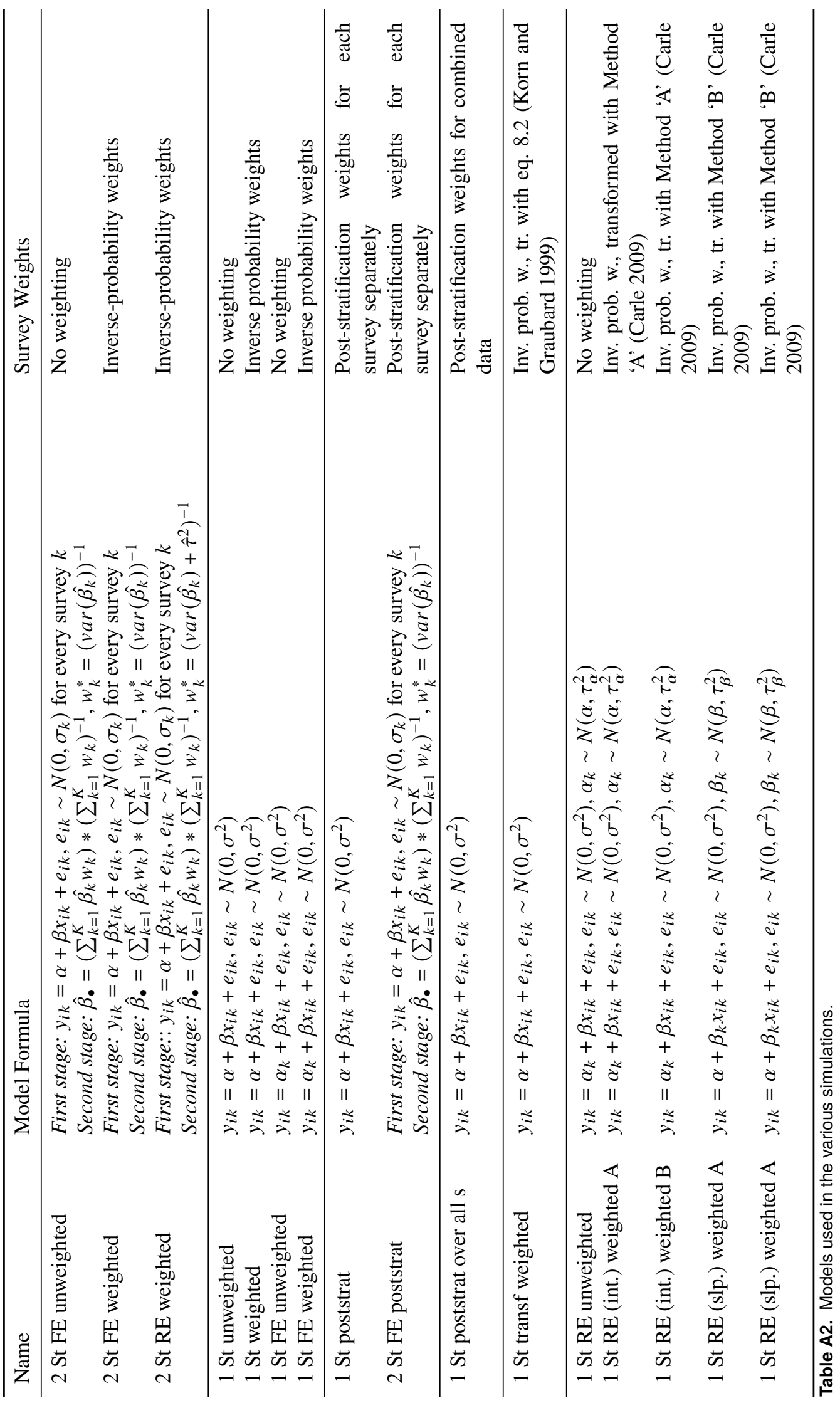




\begin{tabular}{lc}
\hline (Intercept) & $8.67^{* * *}$ \\
& $(0.03)$ \\
Same-sex Partnership & $-1.00^{* *}$ \\
& $(0.35)$ \\
\hline Num. obs. & 7229 \\
\hline${ }^{* * *} p<0.001,{ }^{* *} p<0.01,{ }^{*} p<0.05$
\end{tabular}

Table A3. Data taken from pairfam wave 1, subsample filter: people in partnerships. Subsampling was accounted for in the estimation.

\begin{tabular}{lc}
\hline (Intercept) & $8.39^{* * *}$ \\
& $(0.03)$ \\
Same-sex Partnership & 0.10 \\
& $(0.26)$ \\
\hline Num. obs. & 2686 \\
\hline${ }^{* * *} p<0.001,{ }^{* *} p<0.01,{ }^{*} p<0.05$
\end{tabular}

Table A4. Data taken from GGS wave 2, subsample filter: people in partnerships. Subsampling was accounted for in the estimation.

\begin{tabular}{lc}
\hline (Intercept) & $7.85^{* * *}$ \\
& $(0.04)$ \\
Same-sex Partnership & $-0.73^{*}$ \\
& $(0.35)$ \\
\hline Num. obs. & 7664 \\
\hline${ }^{* * *} p<0.001,{ }^{* *} p<0.01,{ }^{*} p<0.05$
\end{tabular}

Table A5. Data taken from SOEP wave "y", subsample filter: people in partnerships. Subsampling was accounted for in the estimation. 